\title{
Bilayer $\mathrm{SnS}_{2}$ : Tunable stacking sequence by charging and loading pressure
}

\author{
C. Bacaksiz, ${ }^{1}$ S. Cahangirov, ${ }^{2}$ A. Rubio, ${ }^{3,4}$ R. T. Senger, ${ }^{1}$ F. M. Peeters, ${ }^{5}$ and H. Sahin ${ }^{5}$ \\ ${ }^{1}$ Department of Physics, Izmir Institute of Technology, 35430 Izmir, Turkey \\ ${ }^{2}$ UNAM-National Nanotechnology Research Center, Bilkent University, 06800 Ankara, Turkey \\ ${ }^{3}$ Nano-Bio Spectroscopy Group and ETSF, Departamento de Física de Materiales, Universidad del Pais Vasco, \\ CFM CSIC-UPV/EHU-MPC and DIPC, 20018 San Sebastian, Spain \\ ${ }^{4}$ Max Planck Institute for the Structure and Dynamics of Matter and Center for Free-Electron Laser Science, \\ Luruper Chaussee 149, 22761 Hamburg, Germany \\ ${ }^{5}$ Department of Physics, University of Antwerp, Groenenborgerlaan 171, 2020 Antwerp, Belgium
}

(Received 13 January 2016; published 3 March 2016)

\begin{abstract}
Employing density functional theory-based methods, we investigate monolayer and bilayer structures of hexagonal $\mathrm{SnS}_{2}$, which is a recently synthesized monolayer metal dichalcogenide. Comparison of the $1 \mathrm{H}$ and $1 T$ phases of monolayer $\mathrm{SnS}_{2}$ confirms the ground state to be the $1 T$ phase. In its bilayer structure we examine different stacking configurations of the two layers. It is found that the interlayer coupling in bilayer $\mathrm{SnS}_{2}$ is weaker than that of typical transition-metal dichalcogenides so that alternative stacking orders have similar structural parameters and they are separated with low energy barriers. A possible signature of the stacking order in the $\mathrm{SnS}_{2}$ bilayer has been sought in the calculated absorbance and reflectivity spectra. We also study the effects of the external electric field, charging, and loading pressure on the characteristic properties of bilayer $\mathrm{SnS}_{2}$. It is found that (i) the electric field increases the coupling between the layers at its preferred stacking order, so the barrier height increases, (ii) the bang gap value can be tuned by the external E field and under sufficient $\mathrm{E}$ field, the bilayer $\mathrm{SnS}_{2}$ can become a semimetal, (iii) the most favorable stacking order can be switched by charging, and (iv) a loading pressure exceeding $3 \mathrm{GPa}$ changes the stacking order. The E-field tunable band gap and easily tunable stacking sequence of $\mathrm{SnS}_{2}$ layers make this $2 \mathrm{D}$ crystal structure a good candidate for field effect transistor and nanoscale lubricant applications.
\end{abstract}

DOI: 10.1103/PhysRevB.93.125403

\section{INTRODUCTION}

Ultrathin materials [1], the study of which was boosted after the synthesis of graphene [2], have attracted considerable interest due to their remarkable physical properties [3,4]. Graphene has extraordinary mechanical [5] and optical [6] properties. However, due to the lack of a band gap in graphene [7], exploring other two-dimensional (2D) materials with a band gap became important for several applications. In this respect, synthesis and the theoretical prediction of many other $2 \mathrm{D}$ materials have been achieved, such as silicene [8,9], germanene [8,10-12], stanene [13,14], transition-metal dichalcogenides (TMDs) [15-22], and hexagonal structures of III-V binary compounds (e.g., $h$-BN, $h$-AlN) [23-27]. The atomic scale of thickness of these materials led to new physical insights which suggest that possible other 2D materials may exhibit novel properties. In addition, the need for a wide range of materials for device technology makes the discovery of new layered materials essential.

In regard to the search for new graphene-like or TMD-like 2D material, Sn dichalcogenides are a good candidate because of their vdW-linked lamellar crystal structure and energy band gap which is in the visible frequency region. As a member of this family, tin disulfide $\left(\mathrm{SnS}_{2}\right)$ was previously investigated in the bulk form for various applications [28-34]. After the emergence of novel 2D materials and improved production methods such as chemical vapor deposition and chemical and mechanical exfoliation, thinner structures of $\mathrm{SnS}_{2}$ were synthesized for different applications. For example, a few nanometers thick hexagonal $\mathrm{SnS}_{2}$ was used for lithium storage in battery applications [35-38]. To enhance the electrochemi- cal performance, composite forms of $\mathrm{SnS}_{2}$ with graphene were examined [39-43]. Single- and few-layer $\mathrm{SnS}_{2}$ were also used to fabricate a field effect transistor [44-46]. Moreover, the photocatalytic character of single- and few-layer $\mathrm{SnS}_{2}$ was shown in different studies which is directly related to the optical properties of hexagonal $\mathrm{SnS}_{2}$ [47-50]. Furthermore, the $\mathrm{SnS}_{2}$ nanosheet was studied for photosensitive field emission and photodetector applications $[51,52]$.

Recently, Zhang et al. demonstrated that the photoluminescence spectra of $\mathrm{SnS}_{2}$ and $\mathrm{MoS}_{2}$ show additional features when they form a van der Waals heterostructure which is important for the engineering of their electronic and optical properties [53]. Huang et al. investigated the synthesis, characterization, and electronic properties of $\mathrm{SnS}_{2}$, from bulk to monolayer [54]. More recently, Su et al. reported that hexagonal $\mathrm{SnS}_{2}$ is a suitable material for photodetection applications with fast photocurrent response time $\sim 5 \mu \mathrm{s}$ [55]. In addition to these, Ahn et al. successfully synthesized hexagonal $\mathrm{SnS}_{2}$ and orthorhombic $\mathrm{SnS}$ as a polymorphic $2 \mathrm{D}$ heterostructure [56].

Although there are a few computational works on singlelayer hexagonal $\mathrm{SnS}_{2}$ [57,58], comprehensive investigation of electronic and optical properties of its monolayer and bilayer crystal structures are still lacking. Therefore, in this study we concentrate on the monolayer and bilayer forms of hexagonal $\mathrm{SnS}_{2}$. The structural parameters, electronic properties, and optical response of these materials are investigated using $a b$ initio methods. In addition, from the calculated absorbance or reflectivity spectra, the optical signatures which allow one to characterize the structural phase or the stacking order of the $\mathrm{SnS}_{2}$ layers were sought. Furthermore, we investigate the 
effects of an applied perpendicular electric field, charging, and loading pressure on the characteristic properties of bilayer $\mathrm{SnS}_{2}$.

The paper is organized as follows: In Sec. II we give details of our computational methodology. An overview of the structural phases and the electronic and optical properties of monolayer hexagonal $\mathrm{SnS}_{2}$ are presented in Sec. III. In Sec. IV different stacking orders of bilayer $\mathrm{SnS}_{2}$ in the $T$ phase are investigated in detail. The effect of the external electric field, charging, and loading pressure on the bilayer system are studied. Finally, we present our conclusion in Sec. V.

\section{COMPUTATIONAL METHODOLOGY}

Our investigations of the structural, electronic, and optical properties of layered $\mathrm{SnS}_{2}$ were performed using the Vienna $a b$ initio Simulation Package (VASP) [59-61], which is based on density functional theory (DFT). The VASP code solves the Kohn-Sham equations for a system with periodic boundary conditions using iteratively a plane-wave basis set. The Perdew-Burke-Ernzerhof (PBE) form of the generalized gradient approximation (GGA) [62] was adopted to describe electron exchange and correlation. The hybrid DFT-HSE06 functional [63] on top of GGA was used for a more accurate estimation of the band gap, as compared to GGA which usually underestimates the band gap of semiconducting systems. The spin-orbit interaction, which is essential for the TMDs, was included in the calculations. The interlayer interaction is dominated by the vdW forces for such layered materials, which was taken into account by using the DFT-D2 method of Grimme [64,65]. To obtain the charge distribution of the configuration, a Bader charge analysis is used [66,67]. The work function which is defined as the energy difference between the valance band maximum (VBM) and the vacuum level is given in the tables.

Structural optimizations were performed with the following parameters. The kinetic energy cutoff of the plane-wave basis set was $500 \mathrm{eV}$ in all calculations. The total energy difference between the sequential steps in the iterations was taken as $10^{-5} \mathrm{eV}$ as convergence criterion. The convergence for the Hellmann-Feynman force in each unit cell was taken to be $10^{-4} \mathrm{eV} / \AA$. Gaussian smearing of $0.05 \mathrm{eV}$ was used and the pressures on the unit cell were decreased to a value of less then $1.0 \mathrm{kB}$ in all three directions. For the determination of accurate charge densities, Brillouin zone integration was performed using a $35 \times 35 \times 1 \quad \Gamma$-centered mesh for the primitive unit cell. To avoid interactions between adjacent $\mathrm{SnS}_{2}$ monolayers and few-layer systems, our calculations were performed with a large unit cell including $16 \AA$ vacuum space.

In addition, the absorbance and the reflectivity of the previously optimized structures were investigated with and without spin-orbit interaction and also including HSE06 on top of GGA. A $\Gamma$-centered $k$-point sampling of $70 \times 70 \times 1$ was used for monolayer systems. Because of the computational burden, $k$-point sampling was reduced to $35 \times 35 \times 1$ for the bilayer systems, and $21 \times 21 \times 1$ for the calculations that include the HSE06 hybrid functional and spin-orbit interaction. The calculated dielectric function provides us with the optical quantities such as the frequency-dependent absorbance $A(\omega)$ and the Fresnel reflectivity $R(\omega)$ through the formulas

$$
\begin{aligned}
& A(\omega)=\frac{\omega}{c} L \operatorname{Im}[\epsilon(\omega)], \\
& R(\omega)=\left|\frac{\sqrt{\epsilon(\omega)}+1}{\sqrt{\epsilon(\omega)}-1}\right|^{2},
\end{aligned}
$$

where the dielectric function is defined as $\epsilon(\omega)=\epsilon_{1}(\omega)+$ $i \epsilon_{2}(\omega)$ and $\omega$ is the frequency, $c$ is the speed of light, and $L$ is the unit cell length in the perpendicular direction,

\section{III. $H$ AND $T$ PHASES OF SINGLE-LAYER $\mathrm{SnS}_{2}$}

Monolayer $\mathrm{SnS}_{2}$ possesses two different phases, $1 T$ and $1 H$, as shown in Fig. 1. Both phases have three trigonal subplanes where the $\mathrm{Sn}$ subplane is sandwiched by two $\mathrm{S}$ subplanes. The $1 T$ phase is a member of the $P \overline{3} m 2$ space group where subplanes of it are $A B C$ stacked. The $1 H$ is a member of the $P \overline{6} m 2$ space group where subplanes of it are $A B A$ stacked. The lattice vectors of both phases are $\mathbf{v}_{1}=a\left(\frac{1}{2}, \frac{\sqrt{3}}{2}, 0\right), \mathbf{v}_{2}=a\left(\frac{1}{2},-\frac{\sqrt{3}}{2}, 0\right)$, where $\left|\mathbf{v}_{1}\right|=\left|\mathbf{v}_{2}\right|$ and $a$ is the lattice constant. The atomic coordinates of the $1 T$ phase are $\left(\frac{\left|v_{1}\right|}{2}, \frac{\left|v_{1}\right|}{2}, 0\right),\left(\frac{\left|v_{1}\right|}{6}, \frac{\left|v_{1}\right|}{6}, \frac{c}{2}\right)$, and $\left(\frac{5\left|v_{1}\right|}{6}, \frac{5\left|v_{1}\right|}{6},-\frac{c}{2}\right)$ for the $\mathrm{Sn}$ atom and the $\mathrm{S}$ atoms, respectively, where $c$ is the distance between the subplanes of $\mathrm{S}$ atoms. The atomic coordinates of the $1 H$ phase are given as $\left(\frac{\left|v_{1}\right|}{3}, \frac{\left|v_{1}\right|}{3}, 0\right),\left(\frac{2\left|v_{1}\right|}{3}, \frac{2\left|v_{1}\right|}{3}, \frac{c}{2}\right)$, and $\left(\frac{2\left|v_{1}\right|}{3}, \frac{2\left|v_{1}\right|}{3},-\frac{c}{2}\right)$.

We obtained the lattice constants of $3.68 \AA$ and $3.60 \AA$ for $1 T$ and $1 H$, respectively. The corresponding $\mathrm{Sn}-\mathrm{S}$ bond lengths $\left(d_{\text {Sn-S }}\right)$ are $2.59 \AA$ and $2.63 \AA$, which are given in Table I. The energy difference between the $1 T$ and $1 H$ phases is $875 \mathrm{meV}$ per unit cell which shows that the formation of the $1 H$ phase is less favorable than $1 T$. The cohesive energies of the $1 T$ and $1 H$ phases are $3.79 \mathrm{eV}$ and $3.49 \mathrm{eV}$, respectively. These results are consistent with the previous results which find the $1 T$ phase the most favorable form of the monolayer. For both phases, $0.7 e^{-}$is donated to each $\mathrm{S}$ atom by the $\mathrm{Sn}$ atom. This charge transfer is larger as compared to $\mathrm{MoS}_{2}$ in which Mo donates $0.5 e^{-}$to each $\mathrm{S}$. In addition, the work functions $(\Phi)$ of the phases are $7.54 \mathrm{eV}$ and $6.19 \mathrm{eV}$. These work function values are larger than those of graphene and bilayer graphene $(\sim 4.6$ and $\sim 4.7 \mathrm{eV}$ [68], respectively) and of single- and few-layer $\mathrm{MoS}_{2}(\sim 5.4 \mathrm{eV}[69])$.

Band structures of the $1 T$ and $1 H$ phases based on GGA including spin-orbit coupling (SOC) and the HSE06 hybrid functional are given in Fig. 1. The $1 T$ phase of the $\mathrm{SnS}_{2}$ monolayer has an indirect band gap where the VBM is between the $\Gamma$ and $M$ points and the conduction band minimum (CBM) is at the $M$ point. As given in Table I, the band gap of the $1 T$ phase is $1.58 \mathrm{eV}$ within GGA+SOC and $2.40 \mathrm{eV}$ within GGA+HSE06. The $1 H$ phase also has an indirect band gap where the VBM is at the $\Gamma$ point and the CBM is at the $M$ point. The band gap values are $0.78 \mathrm{eV}$ within $\mathrm{GGA}+\mathrm{SOC}$ and $1.58 \mathrm{eV}$ within GGA+HSE06. In Fig. 1, band-decomposed charge densities of the valance and conduction band edges are also given. For both phases, the charge density of the VBM is dominated mainly by the $p_{x}$ and $p_{y}$ orbitals of the $\mathrm{S}$ atom. On 
(a)
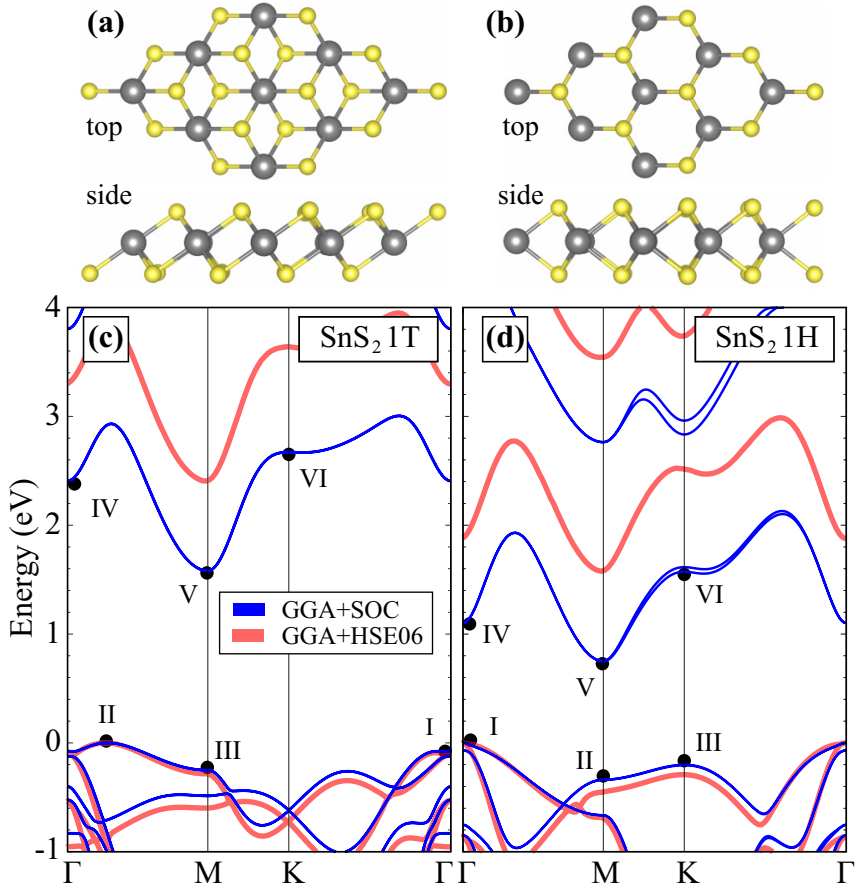

I
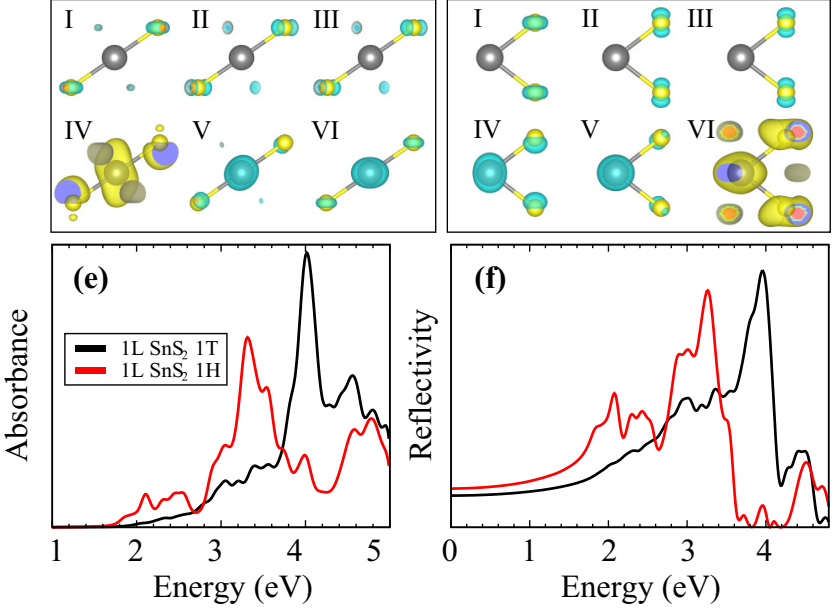

FIG. 1. (a) and (b) illustrate the structure of monolayer $1 T$ and $1 \mathrm{HSnS}_{2}$, respectively. (c) and (d) are the band structures of $1 T$ and $1 \mathrm{HSnS}_{2}$. The blue curves and the red curves are for GGA+SOC and GGA+HSE06, respectively. The orbital character of the band edges is shown below band dispersions. (e) and (f) are absorbance and the reflectivity of the $1 T$ (black curve) and $1 \mathrm{H} \mathrm{SnS}_{2}$ (red curve).

TABLE I. Calculated parameters for monolayer $\mathrm{SnS}_{2}$ are the lattice constant in the lateral direction, $a$; the distance between the subplanes of $\mathrm{S}, c$; the intralayer atomic distance, $d_{\mathrm{Sn}-\mathrm{S}}$; the charge transfer from $S n$ to $S$ atom, $\Delta \rho$; the work function $\Phi$; and the cohesive energy, $E_{c} . E_{g}^{\mathrm{GGA}}$ and $E_{g}^{\mathrm{HSE} 06}$ are the energy band gap values within GGA+SOC and GGA+HSE06, respectively.

\begin{tabular}{lcccccccc}
\hline \hline & $\begin{array}{c}a \\
(\AA)\end{array}$ & $\begin{array}{c}c \\
(\AA)\end{array}$ & $\begin{array}{c}d_{\mathrm{Sn}-\mathrm{S}} \\
(\AA)\end{array}$ & $\begin{array}{c}\Delta \rho \\
\left(e^{-}\right)\end{array}$ & $\begin{array}{c}\Phi \\
(\mathrm{eV})\end{array}$ & $\begin{array}{c}E_{c} \\
(\mathrm{eV})\end{array}$ & $\begin{array}{c}E_{g}^{\mathrm{GGA}} \\
(\mathrm{eV})\end{array}$ & $\begin{array}{c}E_{g}^{\mathrm{HSE06}} \\
(\mathrm{eV})\end{array}$ \\
\hline $1 T-\mathrm{SnS}_{2}$ & 3.68 & 2.96 & 2.59 & 0.7 & 7.53 & 3.79 & 1.58 & 2.40 \\
$1 H-\mathrm{SnS}_{2}$ & 3.60 & 3.23 & 2.63 & 0.7 & 6.19 & 3.49 & 0.78 & 1.58 \\
\hline \hline
\end{tabular}

the other hand, the CBM is dominated by $s$ of $\mathrm{Sn}$ and $p_{z}$ of $S$ orbitals. These orbitals of the band edges are different from that of $\mathrm{MoS}_{2}$ in which the VBM and CBM are composed of $d_{x y}$ and $d_{z^{2}}$, respectively [70].

The effect of the SOC is evident in both the $1 T$ and $1 H$ structures, as shown in Figs. 1(c) and 1(d). In the $1 T$ structure, the splitting is $\sim 50 \mathrm{meV}$ at the highest VB states at the $\Gamma$ point while in the $1 H$ structure the splittings are $\sim 69 \mathrm{meV}$ in the highest VB states at the $\Gamma$ point and $\sim 43 \mathrm{meV}$ in the lowest CB states at the $K$ point. These splittings can be exploited in "valleytronics" applications where the excitations of the electrons with different spin are controlled by the polarization of the incident light. This was recently demonstrated for the TMDs, especially for $\mathrm{MoS}_{2}[71,72]$.

The absorbances and the reflectivities of monolayer $\mathrm{SnS}_{2}$ are also calculated and the energy-dependent plots are given in Figs. 1(e) and 1(f), respectively. The absorbance plot shows that the $1 T$ and $1 H$ phases have different characters. For the $1 T$ phase, absorbance (black) starts at $\sim 1.8 \mathrm{eV}$, and at around $4 \mathrm{eV}$ a peak is found. It has also a local maximum at around $4.5 \mathrm{eV}$. On the other hand, for the $1 H$ phase the absorbance (red) starts at $\sim 1.7 \mathrm{eV}$ and it shows its main peak around $3.2 \mathrm{eV}$, a local maximum around $4.8 \mathrm{eV}$. Since the absorbance spectra of the alternative phases are quite distinguishable, optical absorbance measurements can be a reliable tool for determining the structural phase of monolayer $\mathrm{SnS}_{2}$ samples.

\section{BILAYER $\mathrm{SnS}_{2}$}

Determining or controlling the stacking order of a layered material is important for electronic and optical applications. They can modify the electronic and the optical properties even if the layers are weakly interacting as in van der Waals layered materials. Improvements in synthesis techniques allow researchers to control the stacking order of multilayer structures and synthesis of devices with desired features. Therefore, in this section we investigate properties of bilayer $\mathrm{SnS}_{2}$ starting with the analysis of possible stacking orders.

In Fig. 2, bilayer structures with three different stacking types, their corresponding band diagrams, and the imaginary part of the dielectric functions are given. In the monolayer section, the $1 T$ phase was found to be energetically favorable, and therefore we restrict ourselves to the $1 T$ phase. $A A$ ( $\mathrm{Sn}$ atoms are aligned on $\mathrm{Sn}$ atoms), $A B$ (S atoms are aligned on $\mathrm{Sn}$ atoms), and $A^{\prime} B$ (similar to $A B$ but the bottom layer is upside-down) are considered. Also the $A A^{\prime}$ stacking (not shown) where the $\mathrm{S}$ atoms are aligned on $\mathrm{S}$ is examined, but its total energy is considerably larger as compared to the given three other stacking types. A few meV energy difference was found between the $A A, A^{\prime} B$, and $A B$ stacking orders which are given in Table II where we have set the minimum energy to 0 . All types have the same lattice constant of $3.68 \AA$. The interlayer distances are $2.95 \AA, 2.97 \AA$, and $3.03 \AA$ for the $A A$, $A^{\prime} B$, and $A B$ stacking orders, respectively.

The cohesive energy of bilayer $\mathrm{SnS}_{2}$ in all stacking orders is the same, $3.81 \mathrm{eV}$, which is slightly higher than the monolayer $1 T$ phase. The work functions are also similar where the values are $6.50 \mathrm{eV}, 6.50 \mathrm{eV}$, and $6.58 \mathrm{eV}$ for $A A, A^{\prime} B$, and $A B$, respectively. The work function of the bilayer is smaller than 

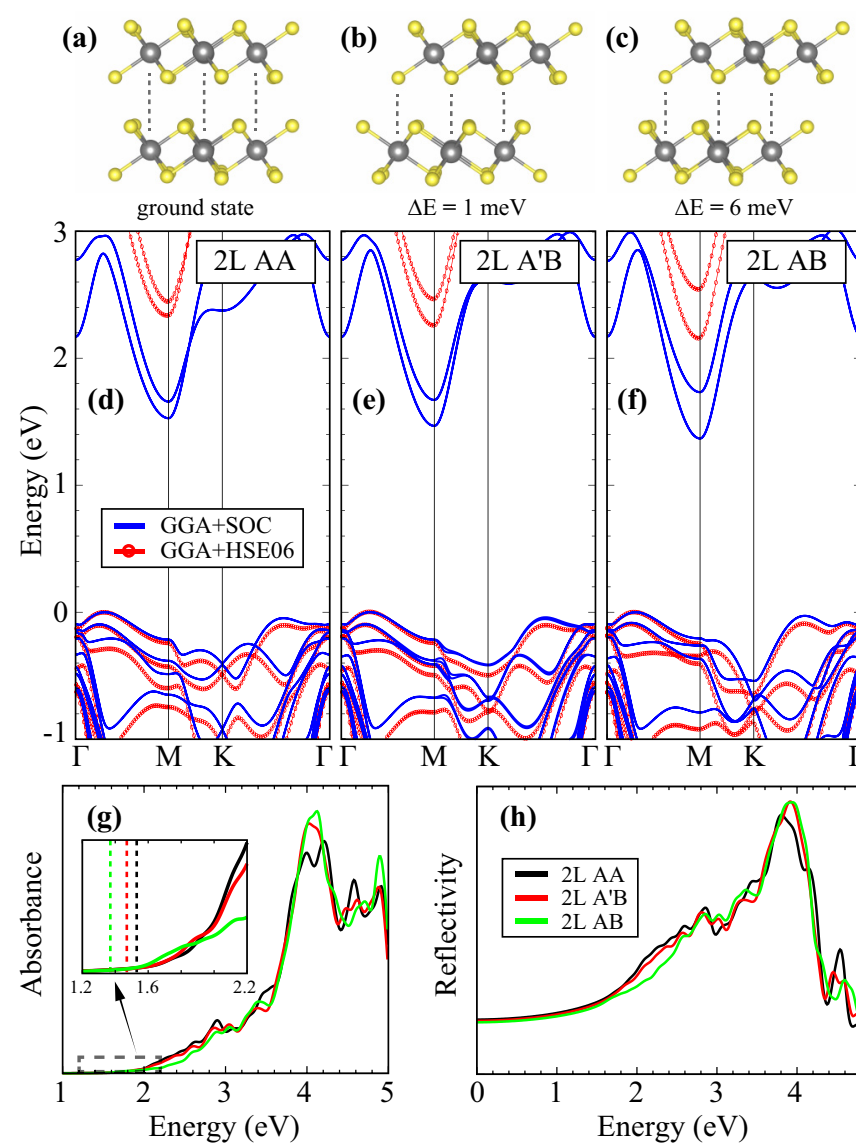

$\Delta \mathrm{E}=1 \mathrm{meV}$ $\Delta \mathrm{E}=6 \mathrm{meV}$
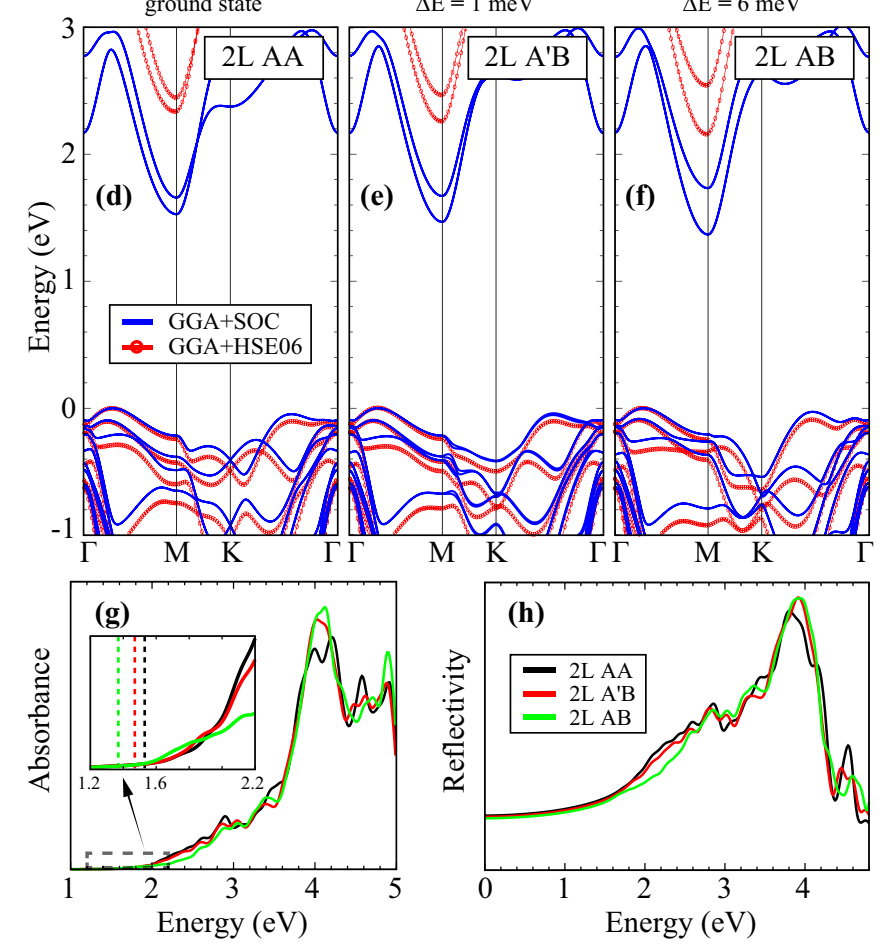

FIG. 2. (a)-(c) are side views of bilayer $\mathrm{SnS}_{2}$, and (d)-(f) are band structures within the GGA+SOC (blue) and GGA+HSE06 (red circles) for $A A, A^{\prime} B$, and $A B$ stackings, respectively. (g) and (h) are the calculated absorbance and reflectivity of the $A A$ (black), $A^{\prime} B$ (red), and $A B$ (green). The vertical lines in the inset of $(\mathrm{g})$ represent the band gap values of the corresponding stacking orders.

that of the $1 T$ monolayer which is in contrast with what was found for graphene and $\mathrm{MoS}_{2}$ [68,69]. Another point is that the interlayer potential energies per $\mathrm{SnS}_{2}$ for the different stacking types are also very close to each other: $38 \mathrm{meV}, 38 \mathrm{meV}$, and $35 \mathrm{meV}$ for $A A, A^{\prime} B$, and $A B$, respectively. This weak interaction is a characteristic feature of van der Waals layered materials, yet these energy values are smaller as compared to

TABLE II. Calculated values for possible stacking types of bilayer $1 T-\mathrm{SnS}_{2}$ of the lattice constant in the lateral direction, $a$; the distance between the $\mathrm{S}$ sublayers of the layers, $d_{L-L}$; the energy difference between the structures per $\mathrm{SnS}_{2}, \Delta E$; interlayer interaction potential per formula, $E_{L-L}$; the work function, $\Phi$; and the cohesive energy, $E_{c} . E_{g}^{\mathrm{GGA}}$ and $E_{g}^{\mathrm{HSE} 06}$ are the energy band gap values within GGA+SOC and GGA+HSE06, respectively.

\begin{tabular}{lcccccccc}
\hline \hline & $\begin{array}{c}a \\
(\AA)\end{array}$ & $\begin{array}{c}d_{L-L} \\
(\AA)\end{array}$ & $\begin{array}{c}\Delta E \\
(\mathrm{meV})\end{array}$ & $\begin{array}{c}E_{L-L} \\
(\mathrm{meV})\end{array}$ & $\begin{array}{c}\Phi \\
(\mathrm{eV})\end{array}$ & $\begin{array}{c}E_{c} \\
(\mathrm{eV})\end{array}$ & $\begin{array}{c}E_{g}^{\mathrm{GGA}} \\
(\mathrm{eV})\end{array}$ & $\begin{array}{c}E_{g}^{\mathrm{HSE} 06} \\
(\mathrm{eV})\end{array}$ \\
\hline$A A$ & 3.68 & 2.95 & 0 & 38 & 6.50 & 3.81 & 1.53 & 2.34 \\
$A^{\prime} B$ & 3.68 & 2.97 & 1 & 38 & 6.50 & 3.81 & 1.47 & 2.27 \\
$A B$ & 3.68 & 3.03 & 6 & 35 & 6.58 & 3.81 & 1.37 & 2.17 \\
\hline \hline
\end{tabular}

graphite (30-55 meV per atom) [73,74] and typical TMDs (74, 107, 90, $126 \mathrm{meV}$ per $M X_{2}$ for $\mathrm{MoS}_{2}, \mathrm{MoSe}_{2}, \mathrm{WS}_{2}, \mathrm{WSe}_{2}$, respectively) [75].

In spite of the weak interlayer interactions, and the similarities of the structural parameters, the $A A, A B$, and $A^{\prime} B$ stacking types possess different band dispersions and band gaps. Although the VBM and the CBM are at the same symmetry points for all stacking orders, the values of the indirect band gaps are different. For $A A$, which is energetically the favorable one, we have a $1.53 \mathrm{eV}$ band gap within GGA and $2.34 \mathrm{eV}$ within HSE06. The band gaps in the $A^{\prime} B$ and the $A B$ stackings are 1.47 and $1.37 \mathrm{eV}$ within GGA and 2.27 and $2.17 \mathrm{eV}$ within HSE06, respectively. It must be emphasized that the band dispersions arising from each bilayer configuration differ especially at the symmetry points $M$ and $K$ which are important for the optical transitions and the excitonic states. For $A A$ stacking, the two $\mathrm{CB}$ edge states at the $M$ point are very close to each other as compared to those of $A^{\prime} B$ and $A B$. On the other hand, the two CB edge states at the $K$ point are significantly different in energy as compared to those of $A^{\prime} B$ and $A B$. The absorbance spectra of the bilayer systems are given in Figs. 2(g) and 2(h). The general trends of the absorbances for all bilayers are similar. The inset of Fig. $2(\mathrm{~g})$ is a zoom at the onset region of the absorbance spectrum. Despite the weak interactions given in Table II, the absorbance spectra provide information on the stacking. In addition, the main peak around $4 \mathrm{eV}$ of the $A A$ stacking displays two distinct peaks while $A^{\prime} B$ and $A B$ have only one peak. Hence, the simple absorbance spectrum carries structural signatures although the structures are energetically very similar.

The weak layer-layer interaction in bilayer $\mathrm{SnS}_{2}$ is also promising for barrierless sliding applications. The sliding potential in the armchair and the zigzag directions are given in Fig. 3. The local and global extrema and the corresponding structural forms are shown. The positions of the upper $\mathrm{S}$ atoms of the bottom layer and lower $S$ atoms of the top layer are responsible for the potential profile. In the case of sliding along

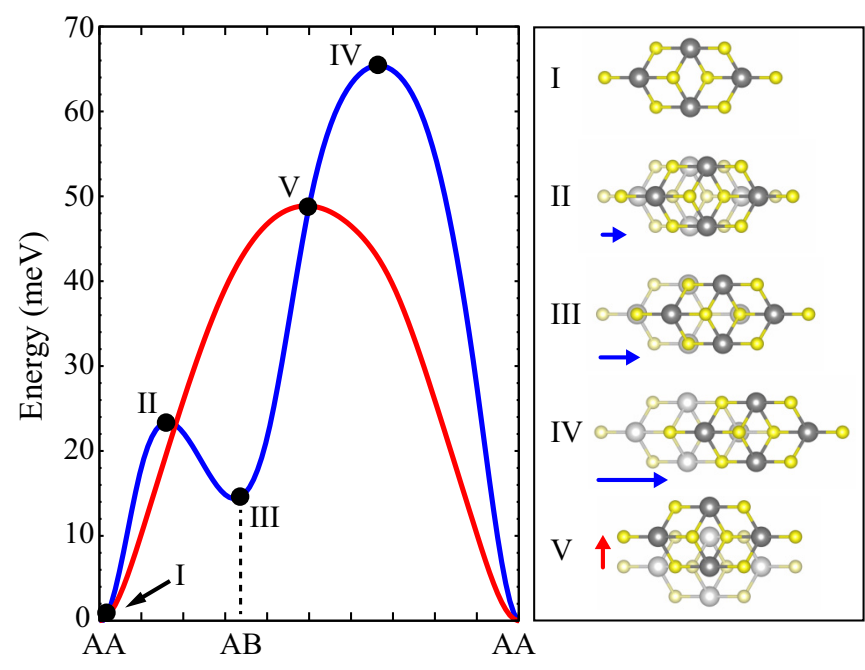

FIG. 3. Left panel: Blue (red) curve indicates the energy difference when the top layer is sliding along the armchair (zigzag) direction. Right panel: The structural forms of the extrema marked on the energy profiles are given. 
the armchair direction the local maximum is seen when the lower $\mathrm{S}$ atoms of the top layer are positioned at the midpoint of the upper $\mathrm{S}$ atoms of the bottom layer. This is followed by a local minimum that corresponds to $A B$ stacking. The highest point of the barrier is $\sim 65 \mathrm{meV}$. This point is also a global maximum where the $\mathrm{S}$ atoms from the top and bottom layers are aligned on top of each other. This energy barrier is very small as compared to that of $\mathrm{MoS}_{2}(\sim 200 \mathrm{meV})$ [20]. In the zigzag direction, the barrier profile results in a symmetric peak with the highest point obtained when the $S$ atoms of the top and bottom layers are closest to each other at the path of sliding. The maximum value of the barrier is $\sim 50 \mathrm{meV}$. This type of barrier is common for the $T$ phase of TMDs. The following subsections investigate how these barrier profiles are modified by electric field, charging, and loading pressure.

\section{A. Effect of external electric field}

In this part, we investigate how the sliding potential is modified under the influence of an external perpendicular electric field (E field). Three different $(0.05,0.15,0.25 \mathrm{~V} / \AA)$ E-field values in the positive $z$ direction (perpendicular to the plane of the bilayer system) are applied. While the energy barrier is conserved, the barrier heights increase as shown in Figs. 4(a) and 4(b). The changes at the $0.05 \mathrm{~V} / \AA$ field is negligible and the profiles are almost the same as in the zero E-field case. In addition, for $A B$ stacking the energy difference between the local minimum and the neighboring local maximum decreases with applied E field. The reason for the changes can be elucidated by analyzing the variations of the charge separation in the system.

In Fig. 4(c), by using the Bader charge analysis technique, the amount of charge difference on the atoms between the $0.25 \mathrm{~V} / \AA$ case and the zero-field case is shown along the sliding path. The solid (dashed) orange curve represents the Sn atoms at the bottom (top) layer. The solid (dashed) blue curve represents the outer $\mathrm{S}$ atom at the bottom (top) layer and the solid (dashed) red curve is for the inner $\mathrm{S}$ atom ( $\mathrm{S}$ atoms between the sublayers of $\mathrm{Sn}$ atoms). The charge configurations seem to be sensitive not only to the E field but also the stacking order of the layers. First, the charge variations of the Sn atoms of the bottom and the top layers are positive which indicates that the E field shifts electron around the Sn atoms. On the other hand, the behaviors of the changes on the $S$ atoms are different according to being at the outer or the inner part of the bilayer system. The outer $\mathrm{S}$ atoms have less electrons under the $E$ field while the inner $S$ atoms attain more electrons. As an exception to these trends, the charge of the $\mathrm{S}$ atoms is not altered much by the E field for the $A A$ and the $A B$ stackings. It needs more detailed analysis.

To clarify the effect of the external $\mathrm{E}$ field on the $A A$ and $A B$ bilayer systems, the total charge density difference between with and without $E$ field for the cross section through the atoms in the unit cell is shown in Fig. 4(d). It seems that the $\mathrm{S}$ atoms are polarized by the E field, but the $\mathrm{Sn}$ atoms experience no significant change. In the case of $0.15 \mathrm{~V} / \AA$, the polarizations are larger at the inner sides of the $\mathrm{S}$ atoms for both $A A$ and $A B$ stackings. In the case of $0.25 \mathrm{~V} / \AA$, the polarization vanishes at the outer $\mathrm{S}$ atoms for $A A$ stacking order. On the
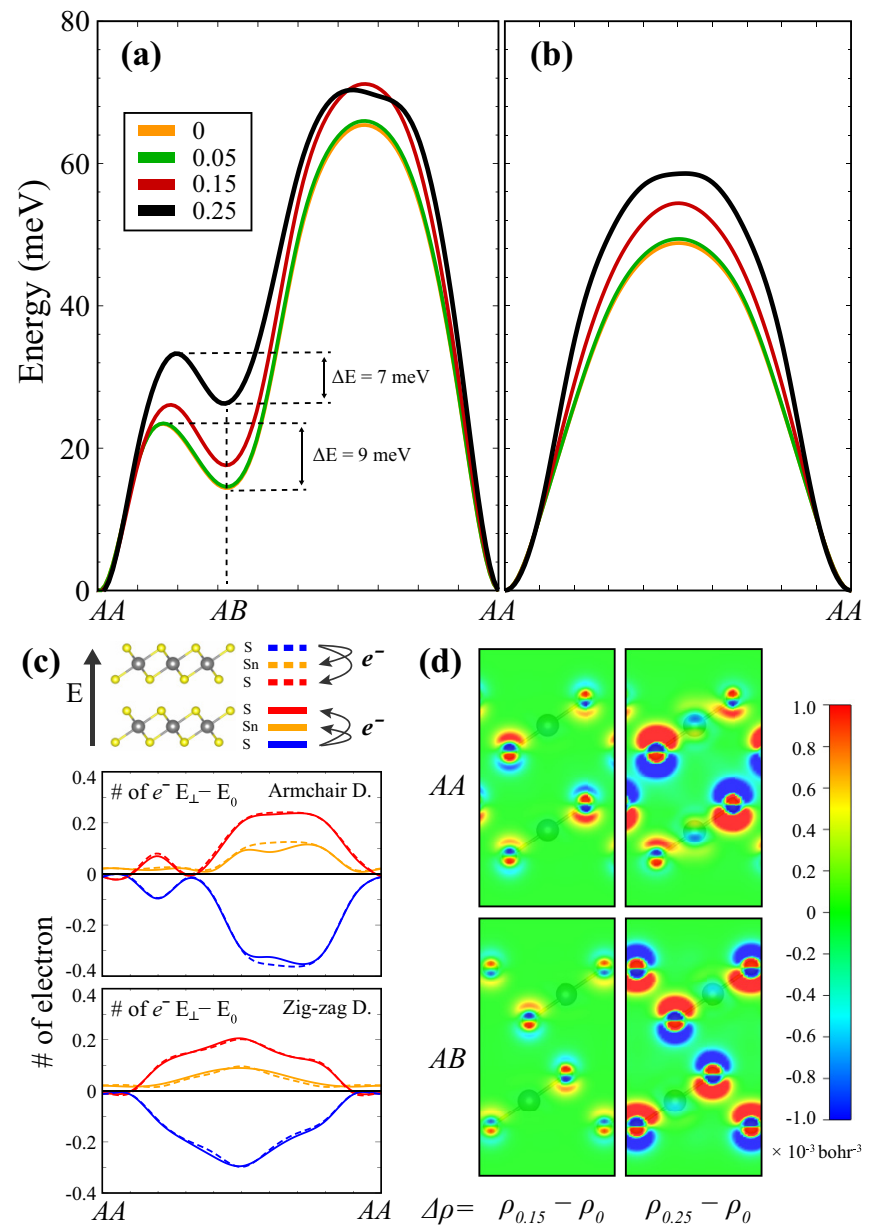

FIG. 4. The plot of the energy barrier in the armchair (a) and the zigzag (b) direction under zero and increasing electric fields. The blue line represents the zero electric field case. The black, green, and red lines are for $0.05,0.15,0.25 \mathrm{~V} / \AA$ A electric field cases, respectively. (c) Electron transfer from outer $\mathrm{S}$ atoms to the inner part (upper) and the total number of electron difference (lower) between the $0.25 \mathrm{~V} / \AA$ electric field case and the zero electric field case on the sliding paths. (d) is the cross section of the total charge density difference between the $0.15,0.25 \mathrm{~V} / \AA$ A electric field cases and the zero field case for both $A A$ and $A B$ stacking orders. The color code of the isosurface values is given.

other hand, the polarization still exists and is enhanced at the inner $\mathrm{S}$ atoms of $A A$ and all $\mathrm{S}$ atoms of $A B$ stacking. More importantly, the number of electrons increases gradually between the layers with electric field strength for both the $A A$ and the $A B$ stackings. This charge accumulation between the layers is consistent with the study of Ramasubramaniam et al. [76], where the $\mathrm{MoS}_{2}$ bilayer is tuned by the external E field and the charge distribution between the layers was gradually enhanced with increasing out-of-plane $\mathrm{E}$ field. According to our results, the perpendicular E field increases the coupling between the $\mathrm{SnS}_{2}$ layers for $A A$ stacking as compared to $A B$.

In addition, the $E$ field dramatically modifies the electronic structure of the bilayer system as shown in Fig. 5. Under the E field, the VBM approaches the $\Gamma$ point while the CBM at the $M$ point drops in energy which means that band gap decreases. 


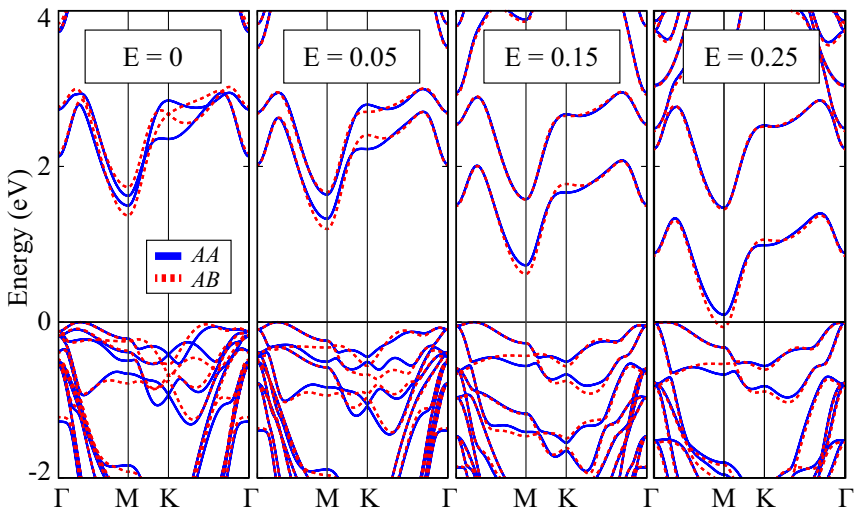

FIG. 5. Energy band dispersions under three different $\mathrm{E}$ fields which are $0.05,0.15$, and $0.25 \mathrm{~V} / \AA$ perpendicular to the plane of the structure. The blue curves are for $A A$ stacking and red curves are for $A B$ stacking.

The drop of band gap with $\mathrm{E}$ field is slower for the $A A$ stacking, so under the $0.25 \mathrm{~V} / \AA \mathrm{E}$ field, the $A B$ become a semimetal while the $A A$ stacked bilayer is a semiconductor with band gap $85 \mathrm{meV}$ within GGA. Although this is the underestimated band gap, the trend of change on electronic structure together with enhanced stacking strength at $A A$ order indicates that the perpendicular E field is a useful method for tuning the band gap of bilayer $\mathrm{SnS}_{2}$, which is needed in a material for the field effect transistor application.

\section{B. Effect of charging}

The sliding-energy barrier can also be tuned by controlling the total charge on the system. In this part, we examine the modifications of the sliding barrier by adding or subtracting electrons (electron or hole doping) to the bilayer. In Figs. 6(a) and 6(b), we show respectively the barrier forms along the armchair and the zigzag directions for four different charging conditions. A positive value of charging refers to extra electrons. First of all, it is interesting that the $0.2 e^{-}$and also the $0.1 e^{-}$(per unit cell) cases result in a minimum energy for the $A B$ stacking instead of $A A$. In addition, the barrier height decreases in all charging conditions and the shape of the barrier differs considerably for the armchair direction. Moreover, the maximum barrier height in the zigzag direction decreases down to $\sim 20 \mathrm{meV}$ which is comparable to the thermal energy at room temperature $(25 \mathrm{meV})$.

The effect of charging can be understood by monitoring the charge localizations for $A A$ and $A B$ stacking. Therefore, in Fig. 6(c), the cross section of the charge density differences between the charged and the bare systems is shown for $A A$ and $A B$ stackings. It is expected that only positively charged regions will appear in the plot for the electron-subtracted system. For the electron-added system, the expectation is the opposite. However the occurrence of both positive and negative regions for each charging case indicates that charging (positive or negative) modifies the distribution of the other electrons. In Fig. 6(c), it is explicitly seen that the inserted charges accumulate to the outer surfaces (red for positive charges and blue for the negative charges) of the bilayer system. In all cases, except the $0.2 e^{-}$added to $A A$ stacking, the electrons
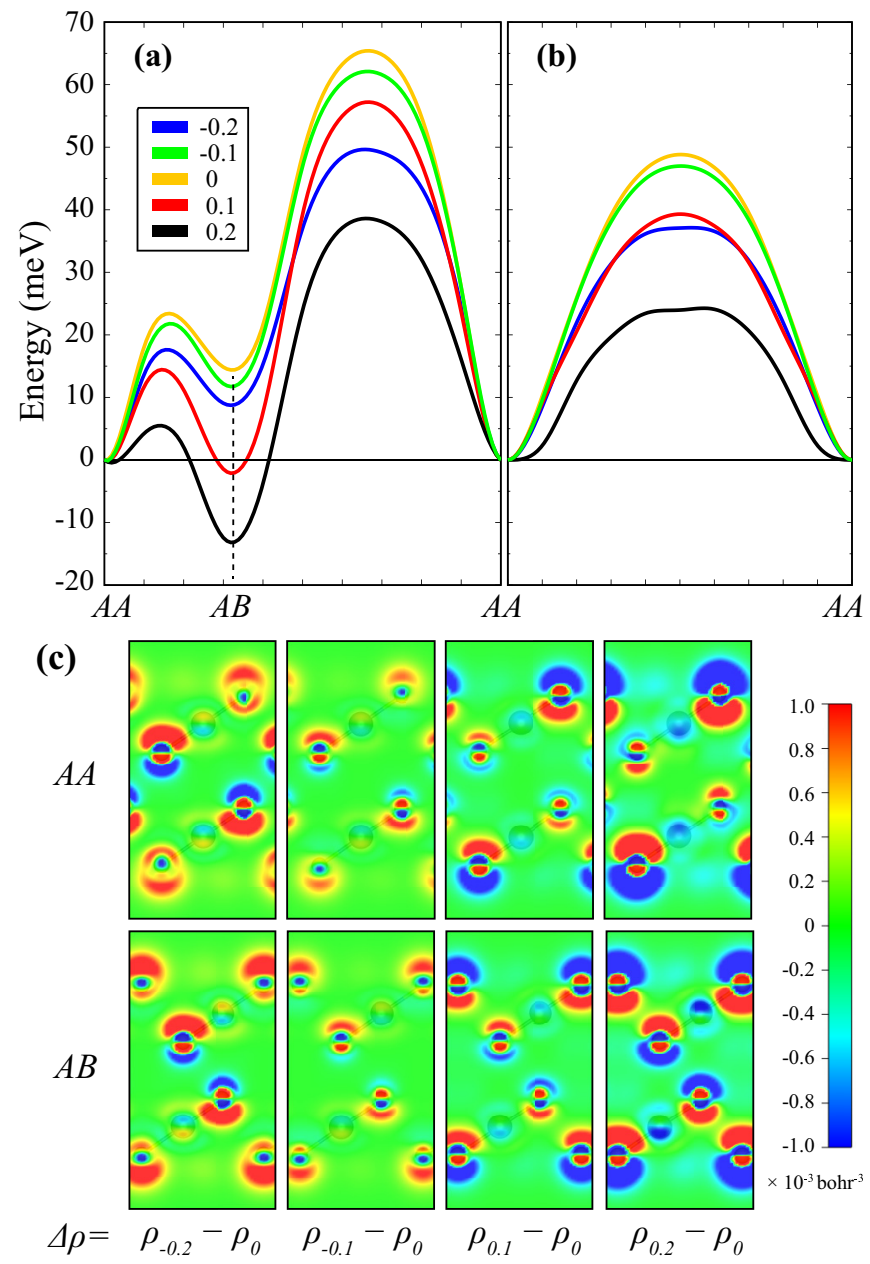

FIG. 6. Plot of the energy barrier for sliding in the armchair (a) and the zigzag (b) direction for four different charging cases and the bare case. Positive value of charing refers to a higher electron density. The blue curves represent the bare case. The green, black, yellow, and red curves are for the charging case of $-0.2,-0.1,0.1$, $0.2 e^{-}$. (c) Cross section of the total charge density difference between the charged cases and the bare case.

(blue region) also accumulate to the region between the layers. When we compare the $A A$ and $A B$ stackings in all charging conditions, the lower energy case has always a larger number of electrons (negative charge means blue region) between the layers which indicates that the interactions between the layers have covalent character.

In addition, when electrons are doped, the $s$ orbital of $\mathrm{Sn}$ atoms which mostly construct the CBM are first occupied as shown in Fig. 7. The energy difference between the newly occupied Sn states and the already occupied S states decreases. The change is higher for the $A B$ stacking as compared to $A A$. For the hole-doping case, $p_{x}$ and $p_{y}$ orbitals of $\mathrm{S}$ atoms which are dominant around the Fermi level (VBM) are first occupied. To sum up, both electron and hole doping decreases sliding barrier which makes it easier to modify the stacking order, and for proper value of electron doping, favorable stacking order become $A B$ stacking instead of $A A$. 


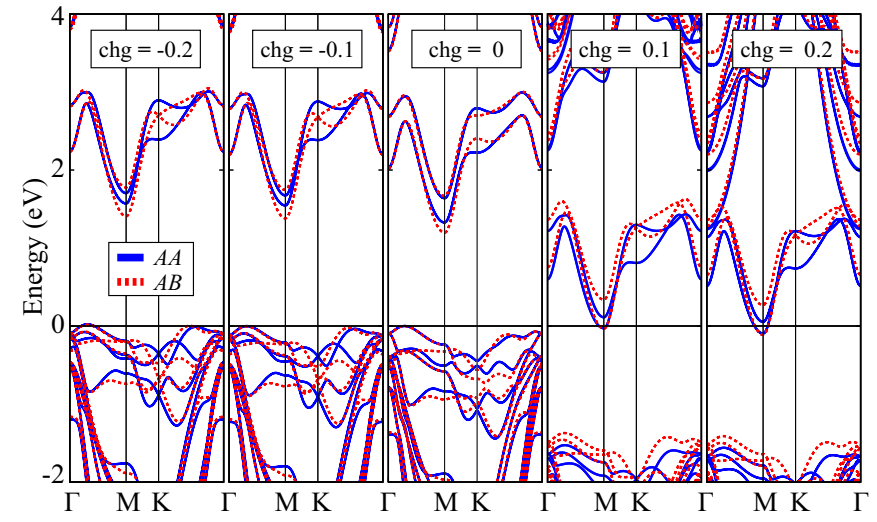

FIG. 7. Energy band dispersions for four different charging conditions which are $-0.2,-0.1,0.1$, and $0.2 e^{-}$. The negative numbers refer to the electron-subtracted (hole-doping) cases and the positive numbers refer the electron-added (electron-doping) cases. The blue curves are for $A A$ stacking and red curves are for $A B$ stacking.

\section{Bilayer under loading pressure}

The energy landscape of bilayers under constant loading pressure is studied for various 2D materials [77]. It is shown that for a given value of the applied pressure, the ratio between intralayer and interlayer interaction is a material property that describes the transition from the stick-slip to the superlubric regime. Here we study the effect of a constant loading pressure on the energy landscape of bilayer $\mathrm{SnS}_{2}$.

The $A A$ and the $A B$ stackings of bilayers, composed of materials like $\mathrm{MoS}_{2}$, have the same energy due to the symmetry of the $1 H$ structure. However, the energy of bilayer $\mathrm{SnS}_{2}$ in $1 T$ is different for $A A$ and $A B$ stackings. This is evident from the previous figures in this section as well as from Fig. 8(a), where we present the constant-height energy landscape of the $\mathrm{SnS}_{2}$ bilayer. To calculate the energy landscape at constant pressure we repeat constant-height scans by lowering the height by $0.2 \AA$ steps. In this way we get the energy for the three-
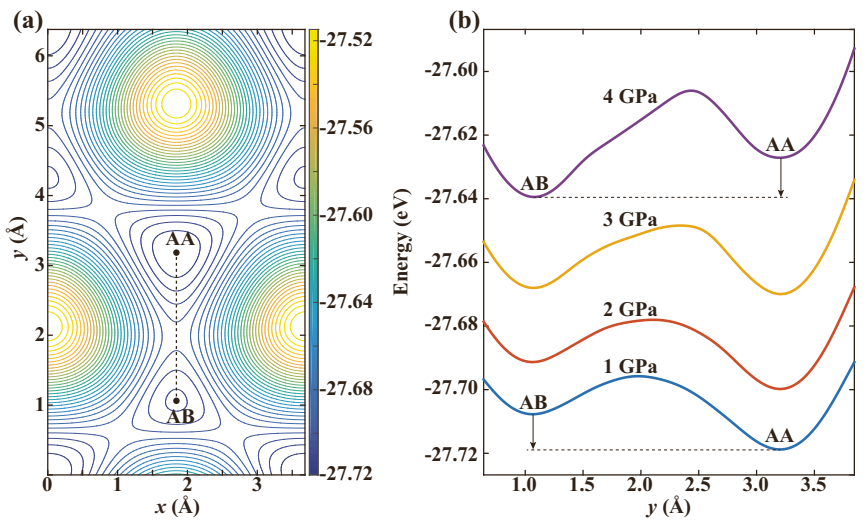

FIG. 8. (a) Constant-height energy landscape of bilayer $\mathrm{SnS}_{2}$. The height is fixed at the value corresponding to the distance between the top and the bottom sulfur atoms when the bilayer is fully relaxed. (b) The constant-pressure energy dependence along the line connecting the $A A$ and the $A B$ stackings. dimensional movement of the layers with respect to each other. We use this data to create the plots presented in Fig. 8(b). Here for a chosen loading pressure we first find the corresponding force in the $z$ direction. Then using spline interpolation we calculate the height that gives this force for each position in the $x y$ plane while moving from $A A$ stacking to $A B$ stacking. Interestingly, as the applied pressure is increased the relative energy difference between $A A$ and $A B$ stackings decreases and become even zero at $3 \mathrm{GPa}$. For pressures exceeding $3 \mathrm{GPa} A B$ stacking becomes more favorable than $A A$ stacking.

\section{CONCLUSION}

Starting from the monolayer, the electronic and the optical properties of bilayer $\mathrm{SnS}_{2}$ are investigated within firstprinciples DFT calculations. We found that the interaction between the layers is weaker than that of $\mathrm{MoS}_{2}$ and other common TMDs. We showed that although the layers interact weakly the energy band gaps and the absorbance spectra could be informative about the stacking type of the bilayer system. The energy barrier for the sliding of one layer over the other is found to be $\sim 65 \mathrm{meV}$ at its maximum, which is also small as compared to $\mathrm{MoS}_{2}$.

The effect of applied E field, charging, and loading pressure on the sliding barrier of bilayer $\mathrm{SnS}_{2}$ were also studied. Under the influence of a perpendicular E field, for the $A A$ stacking which is favorable for the bilayer system, the coupling of the layer strengthens and consequently the sliding barrier height increases. In addition, it is shown that band gap of the bilayer $\mathrm{SnS}_{2}$ can be tuned by perpendicular $\mathrm{E}$ field and under sufficient $\mathrm{E}$ field it can be turned from semiconductor to semimetal. On the other hand, both adding and subtracting electrons decreases the barrier. More significantly, under charging or loading pressure, $A B$ stacking order can become the favorable configuration instead of $A A$ stacking. A tunable band gap makes the 2D crystal of $\mathrm{SnS}_{2}$ a promising material for nanometer-sized field effect transistor applications. Furthermore, due to its easily tunable stacking sequence, layered $\mathrm{SnS}_{2}$ is also a good candidate for nanoscale lubricant applications.

\section{ACKNOWLEDGMENTS}

The calculations were performed at TUBITAK ULAKBIM, High Performance and Grid Computing Center (TR-Grid e-Infrastructure). C.B., H.S., and R.T.S. acknowledge support from TUBITAK Project No. 114F397. H.S. is supported by an FWO Pegasus Marie Curie Fellowship. S.C. and A.R. acknowledge financial support from the Marie Curie grant FP7-PEOPLE-2013-IEF Project No. 628876, the European Research Council (ERC-2010-AdG-267374), and Spanish grant Grupos Consolidados (IT578-13). S.C. acknowledges support from the Scientific and Technological Research Council of Turkey (TUBITAK) under Project No. 115F388. 
[1] K. S. Novoselov, D. Jiang, F. Schedin, T. J. Booth, V. V. Khotkevich, S. V. Morozov, and A. K. Geim, Proc. Natl. Acad. Sci. USA 102, 10451 (2005).

[2] K. S. Novoselov, A. K. Geim, S. V. Morozov, D. Jiang, Y. Zhang, S. V. Dubonos, I. V. Grigorieva, and A. A. Firsov, Science 306, 666 (2004).

[3] S. Z. Butler, S. M. Hollen, L. Cao, Y. Cui, J. A. Gupta, H. R. Gutiérrez, T. F. Heinz, S. S. Hong, J. Huang, A. F. Ismach, E. Johnston-Halperin, M. Kuno, V. V. Plashnitsa, R. D. Robinson, R. S. Ruoff, S. Salahuddin, J. Shan, L. Shi, M. G. Spencer, M. Terrones, W. Windl, and J. E. Goldberger, ACS Nano 7, 2898 (2013).

[4] M. Chhowalla, H. S. Shin, G. Eda, L.-J. Li, K. P. Loh, and H. Zhang, Nat. Chem. 5, 263 (2013).

[5] C. Lee, X. Wei, J. W. Kysar, and J. Hone, Science 321, 385 (2008).

[6] R. R. Nair, P. Blake, A. N. Grigorenko, K. S. Novoselov, T. J. Booth, T. Stauber, N. M. R. Peres, and A. K. Geim, Science 320, 1308 (2008).

[7] S. Y. Zhou, G. H. Gweon, J. Graf, A. V. Fedorov, C. D. Spataru, R. D. Diehl, Y. Kopelevich, D. H. Lee, S. G. Louie, and A. Lanzara, Nat. Phys. 2, 595 (2006).

[8] S. Cahangirov, M. Topsakal, E. Akturk, H. Sahin, and S. Ciraci, Phys. Rev. Lett. 102, 236804 (2009).

[9] A. Kara, H. Enriquez, A. P. Seitsonen, L. C. L. Y. Voon, S. Vizzini, B. Aufray, and Hamid Oughaddou, Surf. Sci. Rep. 67, 1 (2012).

[10] Z. Ni, Q. Liu, K. Tang, J. Zheng, J. Zhou, R. Q. Z. Gao, D. Yu, and J. Lu, Nano Lett. 12, 113 (2012).

[11] M. E. Davila, L. Xian, S. Cahangirov, A. Rubio, and G. Le Lay, New J. Phys. 16, 095002 (2014).

[12] K. Yang, S. Cahangirov, A. Cantarero, A. Rubio, and R. D’Agosta, Phys. Rev. B 89, 125403 (2014).

[13] G. G. Guzman-Verri and L. C. Lew Yan Voon, Phys. Rev. B 76, 075131 (2007).

[14] F. Bechstedt, L. Matthes, P. Gori, and O. Pulci, Appl. Phys. Lett. 100, 261906 (2012)

[15] R. A. Gordon, D. Yang, E. D. Crozier, D. T. Jiang, and R. F. Frindt, Phys. Rev. B 65, 125407 (2002).

[16] J. N. Coleman, M. Lotya, A. O’Neill, S. D. Bergin, P. J. King, U. Khan, K. Young, A. Gaucher, S. De, R. J. Smith, I. V. Shvets, S. K. Arora, J. J. Boland, J. J. Wang, J. F. Donegan, J. C. Grunlan, G. Moriarty, A. Shmeliov, R. J. Nicholls, J. M. Perkins, E. M. Grieveson, K. Theuwissen, D. W. McComb, P. D. Nellist, and V. Nicolosi, Science 331, 568 (2011).

[17] Q. H. Wang, K. Kalantar-Zadeh, A. Kis, J. N. Coleman, and M. S. Strano, Nat. Nanotechnol. 7, 699 (2012).

[18] J. S. Ross, P. Klement, A. M. Jones, N. J. Ghimire, J. Yan, D. G. Mandrus, T. Taniguchi, K. Watanabe, K. Kitamura, W. Yao, D. H. Cobden, and X. Xu, Nat. Nanotechnol. 9, 268 (2014).

[19] H. Sahin, S. Tongay, S. Horzum, W. Fan, J. Zhou, J. Li, J. Wu, and F. M. Peeters, Phys. Rev. B 87, 165409 (2013).

[20] S. Tongay, H. Sahin, C. Ko, A. Luce, W. Fan, K. Liu, J. Zhou, Y.-S. Huang, C.-H. Ho, J. Yan, D. F. Ogletree, S. Aloni, J. Ji, S. Li, J. Li, F. M. Peeters, and J. Wu, Nat. Commun. 5, 3252 (2014).

[21] S. Horzum, D. Cakir, J. Suh, S. Tongay, Y.-S. Huang, C.-H. Ho, J. Wu, H. Sahin, and F. M. Peeters, Phys. Rev. B 89, 155433 (2014).
[22] B. Chen, H. Sahin, A. Suslu, L. Ding, M. I. Bertoni, F. M. Peeters, and S. Tongay, ACS Nano 9, 5326 (2015).

[23] H. Sahin, S. Cahangirov, M. Topsakal, E. Bekaroglu, E. Akturk, R. T. Senger, and S. Ciraci, Phys. Rev. B 80, 155453 (2009).

[24] Q. Wang, Q. Sun, P. Jena, and Y. Kawazoe, ACS Nano 3, 621 (2009).

[25] K. K. Kim, A. Hsu, X. Jia, S. M. Kim, Y. Shi, M. Hofmann, D. Nezich, J. F. Rodriguez-Nieva, M. Dresselhaus, T. Palacios, and J. Kong, Nano Lett. 12, 161 (2012).

[26] P. Tsipas, S. Kassavetis, D. Tsoutsou, E. Xenogiannopoulou, E. Golias, S. A. Giamini, C. Grazianetti, D. Chiappe, A. Molle, M. Fanciulli, and A. Dimoulas, Appl. Phys. Lett. 103, 251605 (2013).

[27] C. Bacaksiz, H. Sahin, H. D. Ozaydin, S. Horzum, R. T. Senger, and F. M. Peeters, Phys. Rev. B 91, 085430 (2015).

[28] B. Fotouhi, A. Katty, and O. Gorochov, J. Electrochem. Soc. 132, 2181 (1985).

[29] B. A. Parkinson, Langmuir 4, 967 (1988).

[30] B. Parkinson, J. Am. Chem. Soc. 112, 7498 (1990).

[31] E. Delawski and B. A. Parkinson, J. Am. Chem. Soc. 114, 1661 (1992).

[32] R. Schlaf, R. D. Louder, O. Lang, C. Pettenkofer, W. Jaegermann, K. W. Nebesny, P. A. Lee, B. A. Parkinson, and N. R. Armstrong, J. Vac. Sci. Technol. A 13, 1761 (1995).

[33] S. K. Panda, A. Antonakos, E. Liarokapis, S. Bhattacharya, and S. Chaudhuri, Mater. Res. Bull. 42, 576 (2007).

[34] D. K. Ma, H. Y. Zhou, J. H. Zhang, and Y. T. Qian, Mater. Chem. Phys. 111, 391 (2008).

[35] T.-J. Kim, C. Kim, D. Son, M. Choi, and B. Park, J. Power Sources 167, 529 (2007).

[36] J.-W. Seo, J.-T. Jang, S.-W. Park, C. Kim, B. Park, and J. Cheon, Adv. Mater. 20, 4269 (2008).

[37] C. Zhai, N. Du, and H. Z. D. Yang, Chem. Commun. 47, 1270 (2011).

[38] J. Ma, D. Lei, L. Mei, X. Duan, Q. Li, T. Wang, and W. Zheng, CrystEngComm 14, 832 (2012).

[39] Z. Jiang, C. Wang, G. Du, Y. J. Zhong, and J. Z. Jiang, J. Mater. Chem. 22, 9494 (2012).

[40] L. Zhuo, Y. Wu, L. Wang, Y. Yu, X. Zhang, and F. Zhao, RSC Adv. 2, 5084 (2012).

[41] B. Qu, C. Ma, G. Ji, C. Xu, J. Xu, Y. S. Meng, T. Wang, and J. Y. Lee, Adv. Mater. 26, 3854 (2014).

[42] T. Zhou, W. Kong Pang, C. Zhang, J. Z. Chen, H. K. Liu, and Z. Guo, ACS Nano 8, 8323 (2014).

[43] Z. X. Huang, Y. Wang, J. I. Wong, and H. Y. Yang, 2D Mater. 2, 024010 (2015).

[44] T. S. Pan, D. De, J. Manongdo, A. M. Guloy, V. G. Hadjiev, Y. Lin, and H. B. Peng, Appl. Phys. Lett. 103, 093108 (2013).

[45] D. De, J. Manongdo, S. See, V. Zhang, A. Guloy, and H. B. Peng, Nanotechnology 24, 025202 (2013).

[46] H. S. Song, S. L. Li, L. Gao, Y. Xu, K. Ueno, J. Tang, Y. B. Cheng, and K. Tsukagoshi, Nanoscale 5, 9666 (2013).

[47] P. Chen, Y. Su, H. Liu, and Y. Wang, ACS Appl. Mater. Interfaces 5, 12073 (2013).

[48] J. Chao, Z. Xie, X. Duan, Y. Dong, Z. Wang, J. Xu, B. Liang, B. Shan, J. Ye, D. Chen, and G. Shen, CrystEngComm 14, 3163 (2012).

[49] Y. Sun, H. Cheng, S. Gao, Z. Sun, Q. Liu, Q. Liu, F. Lei, T. Yao, J. He, S. Wei, and Y. Xie, Angew. Chem., Int. Ed. Engl. 51, 8727 (2012). 
[50] R. Wei, J. Hu, T. Zhou, X. Zhou, J. Liu, and J. Li, Acta Mater. 66, 163 (2014).

[51] P. D. Joshi, C. S. Rout, D. J. Late, and D. S. Joag, J. Vac. Sci. Technol. B 33, 03 C106 (2015).

[52] J. Xia, D. Zhu, L. Wang, B. Huang, X. Huang, and X. M. Meng, Adv. Funct. Mater. 25, 4255 (2015).

[53] X. Zhang, F. Meng, J. R. Christianson, C. Arroyo-Torres, M. A. Lukowski, D. Liang, J. R. Schmidt, and S. Jin, Nano Lett. 14, 3047 (2014).

[54] Y. Huang, E. Sutter, J. T. Sadowski, M. Cotlet, O. L. A. Monti, D. A. Racke, M. R. Neupane, D. Wickramaratne, R. K. Lake, B. A. Parkinson, and P. Sutter, ACS Nano 8, 10743 (2014).

[55] G. Su, V. G. Hadjiev, P. E. Loya, J. Zhang, S. Lei, S. Maharjan, P. Dong, P. M. Ajayan, J. Lou, and H. Peng, Nano Lett. 15, 506 (2015).

[56] J.-H. Ahn, M.-J. Lee, H. Heo, J. H. Sung, K. Kim, H. Hwang, and M.-H. Jo, Nano Lett. 15, 3703 (2015).

[57] H. L. Zhuang and R. G. Hennig, Phys. Rev. B 88, 115314 (2013).

[58] C. Xia, Y. Peng, H. Zhang, T. Wang, S. Wei, and Y. Jia, Phys. Chem. Chem. Phys. 16, 19674 (2014).

[59] G. Kresse and J. Hafner, Phys. Rev. B 47, 558 (1993).

[60] G. Kresse and J. Furthmuller, Phys. Rev. B 54, 11169 (1996).

[61] G. Kresse and D. Joubert, Phys. Rev. B 59, 1758 (1999).

[62] J. P. Perdew, K. Burke, and M. Ernzerhof, Phys. Rev. Lett. 77, 3865 (1996).

[63] J. Heyd, G. E. Scuseria, and M. Ernzerhof, J. Chem. Phys. 118, 8207 (2003).
[64] S. J. Grimme, Comput. Chem. 27, 1787 (2006).

[65] T. Bucko, J. Hafner, S. Lebegue, and J. G. Angyan, J. Phys. Chem. A 114, 11814 (2010).

[66] G. Henkelman, A. Arnaldsson, and H. Jonsson, Comput. Mater. Sci. 36, 354 (2006).

[67] R. F. W. Bader, Atoms in Molecules: A Quantum Theory (Oxford University Press, Oxford, 1990).

[68] Y.-J. Yu, Y. Zhao, S. Ryu, L. E. Brus, K. S. Kim, and P. Kim, Nano Lett. 9, 3430 (2009).

[69] S. Choi, Z. Shaolin, and W. Yang, J. Korean Phys. Soc. 64, 1550 (2014).

[70] J. E. Padilha, H. Peelaers, A. Janotti, and C. G. Van de Walle, Phys. Rev. B 90, 205420 (2014).

[71] K. F. Mak, K. He, J. Shan, and T. F. Heinz, Nat. Nanotechnol. 7, 494 (2012).

[72] T. Cao, G. Wang, W. Han, H. Ye, C. Zhu, J. Shi, Q. Niu, P. Tan, E. Wang, B. Liu, and J. Feng, Nat. Commun. 3, 887 (2012).

[73] Z. Liu, J. Z. Liu, Y. Cheng, Z. Li, L. Wang, and Q. Zheng, Phys. Rev. B 85, 205418 (2012).

[74] X. Chen, F. Tian, C. Persson, W. Duan, and N.-x. Chena, Sci. Rep. 3, 3046 (2013).

[75] J. He, K. Hummer, and C. Franchini, Phys. Rev. B 89, 075409 (2014).

[76] A. Ramasubramaniam, Phys. Rev. B 86, 115409 (2012).

[77] S. Cahangirov, C. Ataca, M. Topsakal, H. Şahin, and S. Ciraci, Phys. Rev. Lett. 108, 126103 (2012). 